\title{
Looking through the FOG: microbiome characterization and lipolytic bacteria isolation from a fatberg site
}

\author{
Elizabeth K. Court ${ }^{1,2, *}$, Roy R. Chaudhuri ${ }^{3}$, Rahul V. Kapoore ${ }^{4,5}$, Raffaella X. Villa ${ }^{6}$, Jagroop Pandhal ${ }^{4}$, Catherine A. Biggs ${ }^{7}$ \\ and Graham P. Stafford ${ }^{1,2, *}$
}

\begin{abstract}
Sewer systems are complex physical, chemical and microbial ecosystems where fats, oils and grease (FOG) present a major problem for sewer management. Their accumulation can lead to blockages ('Fatbergs'), sewer overflows and disruption of downstream wastewater treatment. Further advancements of biological FOG treatments need to be tailored to degrade the FOG, and operate successfully within the sewer environment. In this study we developed a pipeline for isolation of lipolytic strains directly from two FOG blockage sites in the UK, and isolated a range of highly lipolytic bacteria. We selected the five most lipolytic strains using Rhodamine B agar plates and pNP-Fatty acid substrates, with two Serratia spp., two Klebsiella spp. and an environmental Acinetobacter strain that all have the capacity to grow on FOG-based carbon sources. Their genome sequences identified the genetic capacity for fatty acid harvesting (lipases), catabolism and utilization (Fad genes). Furthermore, we performed a preliminary molecular characterization of the microbial community at these sites, showing a diverse community of environmental bacteria at each site, but which did include evidence of sequences related to our isolates. This study provides proof of concept to isolation strategies targeting Fatberg sites to yield candidate strains with bioremediation potential for FOG in the wastewater network. Our work sets the foundation for development of novel bioadditions tailored to the environment with non-pathogenic Acinetobacter identified as a candidate for this purpose.
\end{abstract}

\section{INTRODUCTION}

Sewer systems are an essential component of society, conveying large amounts of domestic and industrial wastes to treatment facilities for safe disposal in dry weather, and additionally safe and effective management of large volumes of rainwater in wet weather. They are complex physical, chemical and microbiological ecosystems. In this context, the entry of fat, oils and grease (FOG) into the wastewater system from both domestic and food service establishment (FSE) sources has become a major problem for the management of wastewater. Indeed, the UK has seen several high-profile blockages caused by FOG deposits in sewers in recent years, phenomena that have now been termed 'fatbergs' [1-4]. Such is the public prominence of these entities, the term fatberg has now entered the English lexicon with inclusion in the Oxford Dictionary in 2015 [5].
The formation of fatbergs is caused by several factors. Firstly, FOGs enter the wastewater system in the form of animal or vegetable fat residues (as triglycerides and free fatty acids), from both domestic, industrial and FSE sources [6-8]. Secondly, these FOGs combine with other material in wastewater systems, such as disposable wipes, to agglomerate forming blockages. In severe cases, the fatbergs can form blockages that are hundreds of metres in length, weigh several metric tonnes and require removal by laborious, dangerous and expensive manual means [9-12]. If left either undetected or untreated, the blockages in sewer networks can lead to sewer overflows, adverse impacts on the environment, for example, through depletion of oxygen in streams, as well as urban flooding [13]. Recent evidence also suggests that this is an increasing problem worldwide - potentially due to rapid population growth and changes in behaviour $[12,14]$. 
While the exact mechanism by which these deposits form is not fully known, a number of factors are thought to contribute. It has been hypothesized that during saponification, free fatty acids (FFAs) combine with calcium and sodium salts in the wastewater to form a nucleation point, leading to solid FOGsoap deposits [8]. It is currently thought that saponification of FFAs, as well as solidification of FOGs in the sewer system, contributes to blockage formation $[1,10,15]$. There is also debate regarding the role of microbes in both the initiation and perpetuation of fatbergs: for example, it has been proposed that bacteria may facilitate the release of calcium salts into the environment - contributing to saponification of FFAs or that, those that release FFA but do not utilize it, can release FFA that causes problems downstream $[1,8,16]$. Another factor is the increased introduction of used cooking oil into wastewater systems, which has a larger amount of FFAs compared to unheated oil and has been shown to be a major cause of the initial nucleation of the FOG [17]. The above factors sit alongside well established culprits of sewer blockages, such as non-flushables like food packaging, condoms and 'flushable' wet wipes [12].

One long proposed potential mitigation approach to FOG accumulation is the use of products composed of sporeproducing single species or off-the-shelf bacterial consortia that are not adapted to the wide environment. These are deployed in the sewer system to speed up the degradation of blockages. If successful, these products have the potential to save time and money for water companies in terms of money spent clearing fatbergs, which has been estimated at $£ 100$ million per year in the UK [18]. However, whilst attractive, the use of lipolytic bacteria that do not utilize FOG, to break down FOGs can lead to the release of FFAs, rather than their consumption or an overall reduction in the sewers. Rather, these FFAs can move downstream from sites of accumulation where they can be subject to saponification and deposit elsewhere [12]. An alternative is the use of more specific fat degrading and consuming bacteria or combinations thereof as part of active FOG degrading microbial consortia that have been considered for Grease Interceptors [19]. Ideally, such a consortium would biodegrade FOG using secreted enzymes, such as lipases, cleaving the ester bond linking FFA with their glycerol backbone breaking down lipids into FFAs, which will then be transported inside the bacterial cells and used as growth substrates. However, the wastewater system is made up of a range of environments, including FSE effluents, sewers, pumping stations and treatment works; all of which have different environmental parameters of temperature, $\mathrm{pH}$, salinity, flow rate, etc. To enable efficient degradation and metabolism of FOG, a consortia with a range of lipases, which target different fatty acids present in FOG, in addition to different rates and abilities to metabolize or assimilate this FOG and that, importantly, are adapted to these different environments would be beneficial.

In the last 20 years, many FOG-degrading products have been tested by water companies in the field and in the laboratory with mixed success, mainly due to a lack of reliability and predictability of the activity of these products in different environments within the wastewater system $[1,19]$. This is at least in part due to their inability to thrive or survive in different parts of the system. One reason may be that in many cases these products are single species or microbial consortia isolated from non-sewer environments, i.e. these are largely lab-adapted lipolytic organisms with long shelf lives. In addition, bioadditions have to work in a different way if targeting the deposits. The fatberg and lipid-rich wastewater are two very different substrates and different products need to be created to treat them.

Our aim in this paper was the isolation and screening of bacteria for lipase activity from real fatberg sites, followed by preliminary characterization of their ability to grow in media mimicking wastewater with a FOG-related carbon source. We also characterized, for the first time, the composition of two exemplar fatberg microbial communities, revealing a broad diversity of organisms. We present our data on a selection of lipolytic isolates and propose that this approach will allow development of FOG-degrading consortia tailored to wastewater environments.

\section{EXPERIMENTAL PROCEDURES Sampling, bacterial growth and Isolation}

Samples were taken from two fatberg sites (site 1 and site 2 ) in the sewer network in collaboration with a UK water company and contracted personnel. Enrichments were performed using rich growth media (Tryptic Soy Broth) and synthetic wastewater (SWWa). The carbon:nitrogen:phosphorus ratio in the SWWa is 100:5:1, which translates to a weight ratio of $800 \mathrm{mgl}^{-1}$ COD: $40 \mathrm{mgl}^{-1}$ ammonia- $\mathrm{N}$ : $8 \mathrm{mgl}^{-1}$ inorganic phosphorus. This consists of the following per litre: $\mathrm{dH}_{2} \mathrm{O}$ (pH 7): $0.0245 \mathrm{~g} \mathrm{~K}_{2} \mathrm{HPO}_{4} ; 0.014 \mathrm{~g} \mathrm{KH}_{2} \mathrm{PO}_{4} ; 0.16 \mathrm{~g} \mathrm{NH}_{4} \mathrm{Cl} ; 0.6 \mathrm{~g}$ $\mathrm{MgSO}_{4} .7 \mathrm{H}_{2} \mathrm{O} ; 0.07 \mathrm{~g} \mathrm{CaCl}_{2} .2 \mathrm{H}_{2} \mathrm{O} ; 0.01 \mathrm{~g}$ EDTA; $2 \mathrm{ml}$ trace mineral. Trace mineral amounts per litre: $1.5 \mathrm{~g} \mathrm{FeCl}_{3} \cdot 6 \mathrm{H}_{2} \mathrm{O}$; $0.15 \mathrm{~g} \mathrm{H}_{3} \mathrm{BO}_{3} ; 0.03 \mathrm{~g} \quad \mathrm{CuSO}_{4} .5 \mathrm{H}_{2} \mathrm{O} ; 0.03 \mathrm{~g} \mathrm{KI} ; 0.12 \mathrm{~g}$ $\mathrm{MnCL}_{2} \cdot 4 \mathrm{H}_{2} \mathrm{O} ; 0.06 \mathrm{Na}_{2} \mathrm{MoI}_{4} \cdot 2 \mathrm{H}_{2} \mathrm{O} ; 0.12 \mathrm{ZnSO}_{4} .7 \mathrm{H}_{2} \mathrm{O} ; 0.15 \mathrm{~g}$ $\mathrm{CoCl}_{2} \cdot 6 \mathrm{H}_{2} \mathrm{O}$ (Karunakaran E., personal correspondence).

The media contained acetic acid $(14 \mathrm{mM})$ as carbon source with the addition of either olive oil $(1 \% \mathrm{v} / \mathrm{v})$ or solid fatberg FOG material $(1 \% \mathrm{w} / \mathrm{v})$, and was incubated with agitation for 3 days at $15-20^{\circ} \mathrm{C}$ before being transferred to new flasks of either TSB or SWWa and enriched for up to 5 days at $15-20^{\circ} \mathrm{C}[20]$. These enrichments were then spread onto both TSB or SWWa agar plates that also contained Rhodamine B (0.0001\%) and olive oil (1\%) and screened for lipolytic activity using lipase assays (see below) and taken forward for analysis [21]. Stocks of isolates from this study were stored in glycerol at $-80^{\circ} \mathrm{C}$.

Growth studies of strains were carried out in synthetic wastewater with either addition of FOG ( $1 \%$ olive oil) or FOG plus acetate $\left(14 \mathrm{mM}, 0.81 \mathrm{mg} \mathrm{ml}^{-1}\right)$. The strains were grown at $25 \mathrm{C}$ in a Tecan Sunrise in 96-well plates with horizontal shaking, $\mathrm{OD}_{600}$ measurements taken at 30 intervals, and all wells having respective control triplicates containing media, media plus oil/ acetate or oil +acetate, which were subtracted 
from the culture positive wells to rule out any emulsification or precipitation effects. Growth studies were carried out in at least triplicate with technical triplicates in each run.

\section{Fatty acid methyl ester profiling}

All chemicals and analytical reagents were of highperformance liquid chromatography grade (Sigma-Aldrich, Dorset, UK) unless stated otherwise. Fatberg samples ( $\sim 5$ to $7 \mathrm{mg}$ ) were weighed followed by direct transesterification as described elsewhere [22-24]. Briefly, $300 \mu \mathrm{l}$ of toluene and $300 \mu \mathrm{l}$ of $0.5 \mathrm{M}$ sodium methoxide were added to the weighed fatberg samples, followed by incubation at $80^{\circ} \mathrm{C}$ for $20 \mathrm{~min}$. After cooling to room temperature, $300 \mu \mathrm{l}$ of $10 \%$ boron trifluoride in methanol was added and the mixture incubated at $80^{\circ} \mathrm{C}$ for $20 \mathrm{~min}$. After cooling to room temperature, $300 \mu \mathrm{l}$ water and $600 \mu \mathrm{l}$ of hexane were added. The mixture was vortexed for $1 \mathrm{~min}$ and centrifuged at $18000 \mathrm{~g}$ at $4^{\circ} \mathrm{C}$ for $10 \mathrm{~min}$. The organic phase was recovered, measured and evaporated to dryness under inert nitrogen gas. The dried fatty acid methyl esters (FAMEs) were reconstituted in $80 \mu \mathrm{l}$ hexane prior to identification and quantification as described elsewhere [24]. In total, five technical replicates were run, among which only the FAMEs identified in three or more replicates were considered true hits. The data was later normalized to dry weight of the samples and FAME's were reported on a percentage basis.

\section{DNA isolation, 16S rRNA sequencing and bioinformatics}

Total DNA extractions from swabs of the sewer wall at the air:liquid interface were resuspended in TE buffer were carried out using DNeasy PowerSoil kit following manufacturer's instructions (Qiagen). DNA quality was assessed using a nanodrop spectrophotometer before being sent to MR DNA (MR DNA, Shallowater, TX, USA) where 16S rRNA V3/V4 variable regions were amplified using primers (341F: CCTACGGGNGGCWGCAG; 806R: GGACTACHVGGGTWTCTAAT; [25]) with barcodes on the forward primer and MiSeq adapters following manufacturer guidelines. Sequencing data were processed using the MR DNA analysis pipeline where the final OTUs were taxonomically classified using BLASTn against a curated database derived from RDPII [26] and NCBI [27]. Heatmaps were generated using Morpheus [28] and PCA analysis performed using METAGENassist [29] PERMANOVA and ANOVA statistical analysis were performed with phyloseq., as implemented in the tool MicrobiomeAnalyst [30,31]. These sequence data have been submitted to the DDBJ/EMBL/GenBank databases under accession number (ERS4556234), while OTU level data and frequencies are present in the Supplementary Material files (available in the online version of this article).

\section{Genome sequencing}

Genomic DNA from isolated lipase producers were carried out using Wizard Genomic DNA Purification Kit (Promega) before sequencing at MicrobesNG, Birmingham. Genomic DNA libraries were prepared using Nextera XT Library Prep
Kit (Illumina, San Diego, USA) using Hamilton Microlab STAR automated handling system, following the manufacturer's protocol with the following modifications: 2 ng DNA was used as input and PCR elongation for $1 \mathrm{~min}$. Pooled libraries were quantified using Kapa Biosystems Library Quantification Kit for Illumina on a Roche light cycler 96 qPCR machine. Libraries were sequenced on the Illumina HiSeq using a $250 \mathrm{bp}$ paired end protocol. Reads were adapter trimmed using Trimmomatic version 0.30 with a sliding window quality cut off of Q15 [32]. De novo assembly was performed on samples using SPAdes version 3.7 [33] and contigs were annotated using Prokka [34]. Table 1 indicates accession numbers at the EMBL database.

For 16S-based phylogeny, all available complete genomes of Serratia, Klebsiella and Acinetobacter were downloaded, and the longest 16S rRNA gene from each was identified using Barrnap [35]. Following initial phylogenetic analysis, the strains most closely related to the five SFB genomes were identified. The $16 \mathrm{~S}$ rRNA sequences were aligned using MUSCLE [36], and redundant sequences and sequences shorter than $90 \%$ of the length of the longest sequence were purged from the alignment. Maximum-likelihood phylogenies of each alignment were constructed with RAxML [37], using the general time reversible (GTR) model of nucleotide substitution with a Gamma model of rate heterogeneity. Then, 100 bootstrap replicates were performed using the RAxML rapid bootstrapping algorithm [38]. The default values were used for all other options. The values of the Gamma distribution shape parameter alpha and the GTR nucleotide substitution rates were estimated from the data. Phylogenies were displayed using UGENE version 40.0, as phylograms with Bootstrap and distances displayed [39]. The closest available reference genome for each sequenced strain was identified using the $16 \mathrm{~S}$ rRNA phylogeny and whole-genome comparisons were performed using NUCmer, part of the MUMmer package, using PATRIC version 3.6.11 (MinHash) [40]; and through MicrobesNG identifying the closest reference genome using Kraken [41, 42]. Genomic DNA analysis and sequence searching was performed using PATRIC and NCBI, while SignalP version 5.0 and SecretomeP version 2.0 were used to screen sequences for signal peptide presence [43-46].

\section{Lipase assays}

Rhodamine B agar plates were used to indicate lipolytic bacterial colonies by the presence of lipid enzymes (lipase/ esterase; [21]. To 11 of autoclaved media agar (NB or SWWa) add $1 \%(\mathrm{w} / \mathrm{v})$ olive oil and $0.1 \mathrm{mg} \mathrm{ml}^{-1}$ Rhodamine B solution with vigorous shaking and the media poured into agar plates. Bacteria were streaked or spread onto these plates and lipolytic colonies were identified using a UV transilluminator and fluorescent colonies taken forward for further processing.

Lipase activity was also investigated semi-quantitatively using $p$-nitrophenol release from $p$-nitrophenol ester at $410 \mathrm{~nm}$ in a TECAN plate reader (INFINITE 2000). The reaction mixture contained $50 \mathrm{mM}$ Tris- $\mathrm{HCl} \mathrm{pH} 7.5,1 \mathrm{mM} \mathrm{CaCl}_{2}, 0.3 \%(\mathrm{v} / \mathrm{v})$ Triton X-100, $1 \mathrm{mM} \mathrm{p-NPP,} \mathrm{made} \mathrm{fresh} \mathrm{in} \mathrm{every} \mathrm{case,} \mathrm{to} 180 \mathrm{ul}$ 


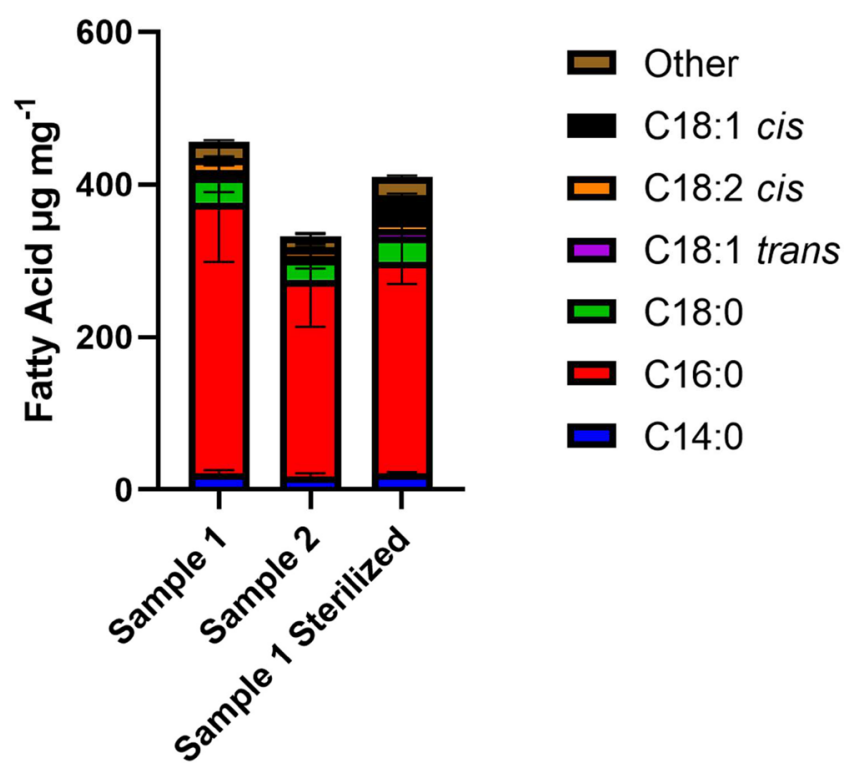

Fig. 1. Bar charts showing the six most abundant fatty acids in two fatberg samples isolated from London sewers ('sample 1' and 'sample 2') and sterilized FOG sample isolated from sample 1 ('sterilized sample 1') as $\mu \mathrm{g} \mathrm{mg}^{-1}$ Fatberg sample. Error bars show standard deviation.

of this, $20 \mathrm{ul}$ of bacterial supernatant was added. This was incubated at room temperature for $30 \mathrm{~min}$ and measuring at $410 \mathrm{~nm}$. In our calculations we used the molar extinction coefficient reported for this substrate previously $\left(17300 \mathrm{M}^{-1}\right.$ $\mathrm{cm}^{-1}$ [47]; and report activity as nmoles pNP released per minute.

\section{Data availability}

Sequence data generated in this study have been deposited to the European Nucleotide Archive (https://www.ebi.ac.uk/ ena) with accession numbers as follows: Serratia marcescens SFB6, ERS4270774; Klebsiella oxytoca SFB9, ERS4270775; Serratia liquefaciens SFB10, ERS4270776; Acinetobacter bouvetii SFB21, ERS4270777; Klebsiella pneumoniae SFB23, ERS4270778; 16S sequencing data, ERS4556234. Raw data for FAME work is in Fig. A.1 and microbiome work is in Fig. A.3.

\section{RESULTS}

\section{Fatty acid profiling of fatberg samples}

In this paper, we began with one aim being to isolate a range of FOG degrading lipolytic strains from fatbergs within the wastewater environment using lipase activity as a screen. As a first step to ensure that our lipase screening methods would target the correct lipolytic profile in comparison to fatberg environments, we determined the lipid profile of solid fatberg samples taken from two separate exemplar Fatberg sites (site 1 and site 2) in London, UK, using gas chromatography-flame ionization detector (GC-FID) by comparison with known fatty acid methyl ester (FAME) standards on five samples from each fatberg. The data reveal that the fatbergs have different overall FOG content at 455.8 $\mu \mathrm{g} \mathrm{mg} \mathrm{m}^{-1}$ and $331.9 \mu \mathrm{g} \mathrm{m}^{-1}$ (Fig. 1, Table S1). However, in both cases the overall profiles (Fig. S1) were similar (not statistically different) with the most abundant fatty acid (FA) being C16 Palmitic acid (average $77 \%$ of FAME present), followed by Myristic (C14,5\%), stearic (C18, 8\%) and linoleic acids (C18:2 cis, 3.8\%). However, analysis of the minor constituent FAs revealed statistically significant differences in the amounts of cis-5,8,11,14,17-Eicosapentaenoic acid (C20:5; 0.776\% : 0.404\% $P=0.0243$ ), Behenic acid (C22; 0.478\%->0.282\% $P=0.000752),(C 24 ; 0.161: 0.112 \%$ $P=0.00050 .7)$ and Nervonic acid (C24: $1 ; 0.200 \%: 0.103 \%$ $P=8.61 \times 10-5)$, however, given their low levels in the samples the importance of this is unclear. As part of our studies, we also sterilized a small portion of solid FOG sample from site 1 (in technical triplicate) via a dry-heat method $\left(160^{\circ} \mathrm{C}, 2 \mathrm{~h}\right)$; the aim here was to assay any changes in the FAMEs profile and establish if the use of these samples in our enrichment and isolation experiments was possible. The heating process caused no significant overall change $(P=0.364$; paired $t$-test $)$ when heat sterilized, however, a significant $(P<0.05)$ increase in oleic acid (C18:1 cis; $\left.0.2->8 \% P=6.4 \times 10^{-5}\right)$ and also in amounts of Arachidonic acid (C20:4n6; 0.006\%->0.058\% $P=0.0182)$ and cis-4,7,10,13,16,19-Docosahexaenoic acid (C22:6n3; 0.008\%->0.079\% $P=0.031$ ). At the same time, the amount of palmitate, the most abundant FA, reduced by $9.16 \%$ but was not statistically significant $(P=0.987)$.

\section{Isolation of lipase-producing bacteria from fatberg samples}

To isolate potential FOG degraders from the blockage sites, wastewater, fatberg and sewer-wall swab samples were first pre-enriched in both a rich broth (Tryptic Soy Broth; TSB) and synthetic wastewater (SWWa) minimal media that contains a range of mineral salts, including acetate $(14 \mathrm{mM}$, $\left.0.81 \mathrm{mgl}^{-1}\right)$, a common wastewater carbon source $[48,49]$, with the addition of either olive oil $(1 \% \mathrm{v} / \mathrm{v})$ or solid FOG sample $(1 \% \mathrm{w} / \mathrm{v})$ and incubated for 3 days before being transferred to a new flask of either TSB or SWWa and enriched for 5 days at $15-20^{\circ} \mathrm{C}[20]$. Importantly this media has a C:N:P ratio of 100:5:1; which has been shown to be effective in environmental and lab studies to aid FOG degradation [50]. These enrichments were then spread onto both TSB and SWWa agar plates that also contained Rhodamine B (0.0001\%) and olive oil (1\%). Olive oil was chosen both for consistency but also due to its composition of oleic acid and linoleic (C18) and palmitic (C16) acid, long chain fatty acids all present in our fatberg (and heated) samples (Fig. 1) [21, 51].

In this method, lipolytic organisms cleave the FOG substrate, releasing FFA that then reacts with Rhodamine $B$, resulting in fluorescence that can be observed via ultraviolet illumination of the agar plates, with lipolysis appearing as orange fluorescent colonies (Fig. S2) [21, 51]. Using this method, colonies were identified with potential high lipolytic activity observed via the production of an orange halo on UV illumination and passaged on SWWa FOG and TSB plates. All five selected strains produced strong haloes on RhB agar 


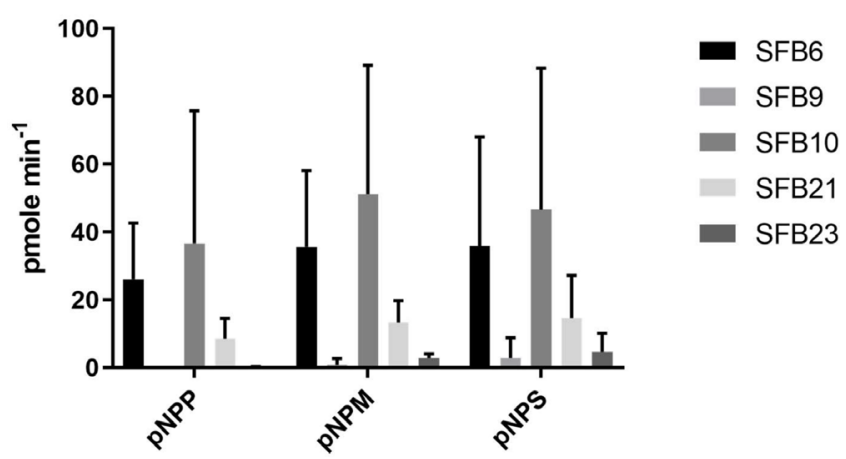

Fig. 2. Bar chart to show the rate of activity in the presence of pNPPPalmitate, -Myristate and -Stearate. Average of three cultures with SEM shown.

(Fig. S2). In order to assess whether these strains contained secreted lipase activity they were grown in nutrient broth with $1 \%$ olive oil, and culture supernatants (normalized to cell density) screened for activity against substrates representing the major lipid constituents in the FAME analysis, namely p-nitrophenyl-palmitate ( $\mathrm{pNP}-\mathrm{P})$, pNP-myristate ( $\mathrm{pNP}-\mathrm{M})$ and $\mathrm{pNP}$-stearate (pNP-S). Using a combination of the RhB plates and $\mathrm{pNP}$ assays, we screened several hundred isolates and selected five with high lipase activity first by picking all colonies with $\mathrm{Rh}$-haloes and then screening using the pNPP assay. These five isolates had lipase activity in the range up to 36.6 pmoles $\mathrm{pNP}$ released $\mathrm{min}^{-1}$, with activity against not only Palmitate but also Stearate and myristate (Fig. 2), validating our strategy of prescreening on RhB-oil plates. Of these, three were isolated with pre-enrichment in synthetic wastewater plus oil followed by TSB plus oil (21, 23), whilst two were pre-enriched in TSB plus oil followed by SWWa plus oil enrichment $(6,9,10)$.

All five selected strains, from now on called SFB6, 9, 10, 21 and 23 (SFB: SheffieldFatBerg) displayed activity against pNP substrates. The results, reported in Fig. 2, showed that SFB6 and SFB10 had the highest activity against all substrates $\left(25.8,51.1\right.$ pmoles $\mathrm{min}^{-1}$ released) while SFB21 also had broad activity of 8.56-14.57 pmoles $\mathrm{min}^{-1}$ released for all substrates. Although SFB9 and 23 had clear haloes on RhB plates they exhibited significantly lower lipase activity under the conditions tested. None of the strains displayed a particular preference for any of the substrates over another, with the exception of SFB23, which seemed to prefer Stearate and Myristate over Palmitate.

\section{Genome analysis of isolates}

Using a combination of $16 \mathrm{~S}$ rDNA and whole-genome sequence phylogeny [MicrobesNG (Kraken) and PATRIC (MinHash)], the isolated SFB strains were identified as potential new strains of Serratia marcescens (SFB6), Klebsiella oxytoca (SFB9), S. liquefaciens (SFB10), Acinetobacter bouvetii (SFB21) and K. pneumoniae (SFB23) (Table S3, Fig. S3). Notably, these bacterial isolates are all common environmental organisms that have been reportedly found in soil, water sources and treatment plants. In order to characterize these organisms further, we examined their genome sequences for the presence of potential lipase enzyme encoding and lipid transport and metabolism genes. Using Illumina-based sequencing each genome dataset was assembled into contigs and submitted to the European Nucleotide Archive (ENA; see Table 1 for accession numbers).

All of the five bacterial isolates contain at least one putative lipase encoding gene sequence, (Fig. 3b). Despite the lipolytic activity, SFB23 (K. pneumoniae) does not appear to contain a putative secreted lipase sequence in its genome sequence, implying that it is not exported via a standard secretion signal or that other unknown exported lipases exist in this strain. Of the strains isolated Acinetobacter SFB21 has the highest number of putative lipase sequences, with seven putative lipase genes of which five are predicted to be secreted enzymes (PSORT), with all lipases containing predicted active sites matching those of the GXXX family [52].

The fatty acid degradation pathways and the main proteins involved in the lipid metabolism have been highlighted in Fig. 3a), i.e. to establish the likelihood these organisms can utilize as well as produce FFAs. All isolates contain full putative fatty acid degradation pathways (Fig. 3b) and the predicted genes encoding fatty acid transport ( $f a d L$ and $f a d D$ ), the $\beta$-oxidation pathway for fatty acid metabolism ( $f a d A, f a d B$ and $f a d E$ ) alongside the transcriptional repressor $(f a d R$, whose repression is relieved by fatty acid binding to the protein). All five isolates contained at least one copy of each of the genes apart from A. bouvetii SFB21, which did not contain a recognisable fadR gene - indicating that canonical fatty acid regulated control of gene

Table 1. Summary genome information including accession (Ac) number

\begin{tabular}{|c|c|c|c|c|c|c|}
\hline Isolate & Homology & \#Contigs & No. of Bases & Size (Mb) & $\mathrm{G}+\mathrm{C}$ content $(\%)$ & Ac no. \\
\hline SFB6 & Serratial marcescens & 77 & 332.8 & 5.339 & 59.18 & ERS4270774 \\
\hline SFB9 & Klebsiella oxytoca & 155 & 665.6 & 6.38 & 55.31 & ERS4270775 \\
\hline SFB10 & Serratia liquefaciens & 29 & 290 & 5.2 & 55.36 & ERS4270776 \\
\hline SFB21 & Acinetobacter bouvetti & 69 & 253.8 & 3.46 & 51.24 & ERS4270777 \\
\hline SFB23 & Klebsiella pneumoniae & 47 & 156.4 & 5.42 & 57.22 & ERS4270778 \\
\hline
\end{tabular}




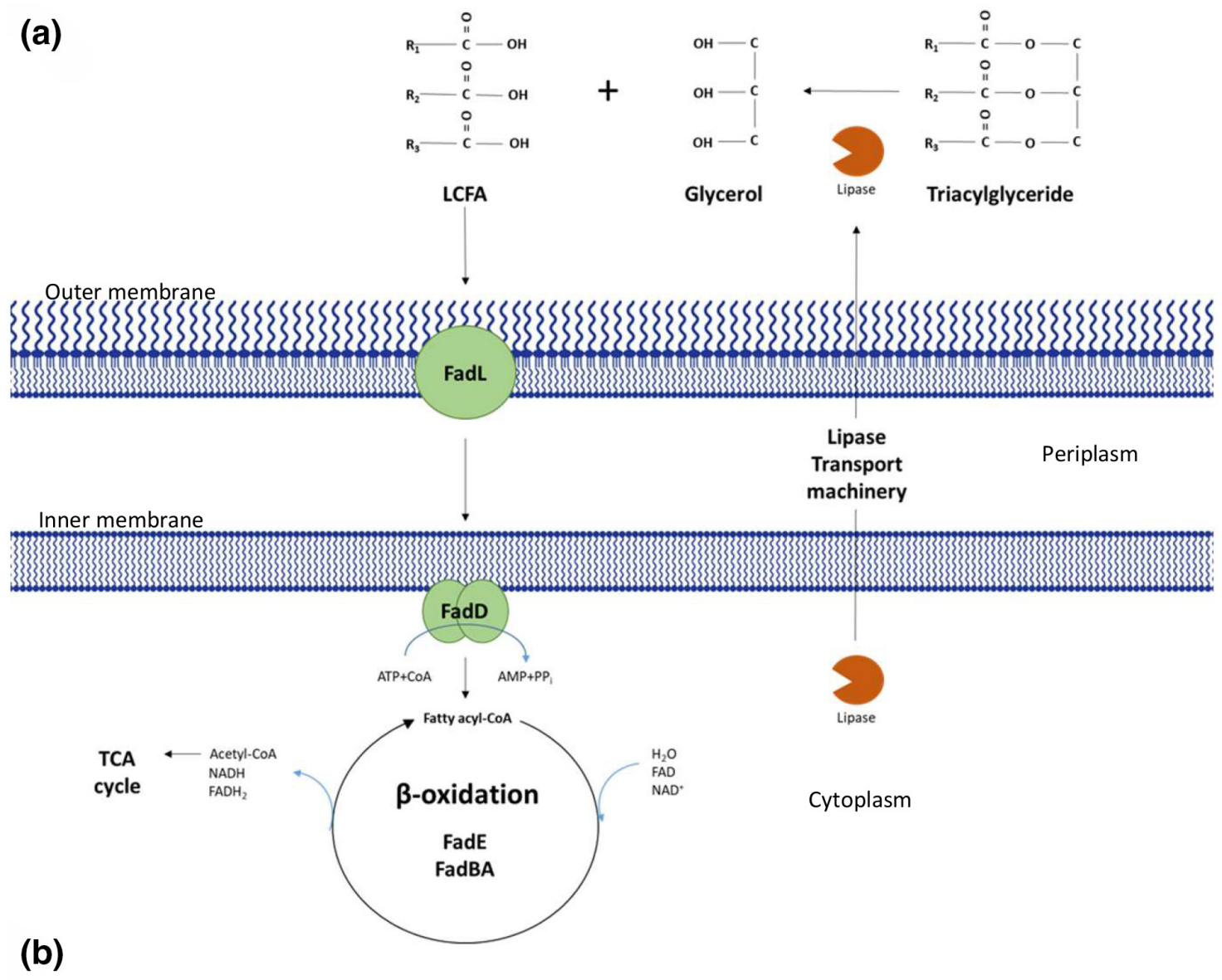

\begin{tabular}{|r|l|l|l|l|l|l|l|l|}
\hline & FadL & FadD & FadE & FadA & FadB & FadR & Lipase & Secreted* \\
\hline S.marcescens S:FB6 & $\bullet$ & $\bullet \bullet \bullet \bullet$ & $\bullet$ & $\bullet$ & $\bullet$ & $\bullet$ & $\bullet \bullet \bullet$ & $\bullet \bullet \bullet$ \\
\hline K.oxytoca S:FB9 & $\bullet \bullet$ & $\bullet \bullet \bullet$ & $\bullet$ & $\bullet$ & $\bullet$ & $\bullet$ & $\bullet \bullet$ & $\bullet \bullet$ \\
\hline S.liquefaciens S:FB10 & $\bullet$ & $\bullet \bullet \bullet \bullet \bullet$ & $\bullet$ & $\bullet$ & $\bullet$ & $\bullet$ & $\bullet \bullet$ & $\bullet \bullet$ \\
\hline A. bouvetii S:FB21 & $\bullet \bullet$ & $\bullet$ & $\bullet \bullet \bullet \bullet \bullet$ & $\bullet$ & $\bullet$ & - & $\bullet \bullet \bullet \bullet \bullet \bullet \bullet$ & $\bullet \bullet \bullet \bullet \bullet \bullet \bullet$ \\
\hline K.pneumonia S:FB23 & $\bullet$ & $\bullet$ & $\bullet$ & $\bullet$ & $\bullet$ & $\bullet$ & $\bullet$ & $\bullet$ \\
\hline
\end{tabular}

Fig. 3. (a) Illustration to show the bacterial FOG catabolism pathway (adapted from [92]). FadL, long-chain fatty acid transport protein; FadD, fatty acid CoA ligase; FadE, acyl-CoA dehydrogenase; FadA, 2-ketoacyl-CoA thiolase; FadB, enoyl-coA hydratase; LCFA, long-chain fatty acid. (b) Summary of homologous genes to bacterial lipid catabolism genes and lipases in SFB isolate strains. Dots correspond to homologous genes in sequence, green represents presence of transport sequence detected. Genomic DNA analysis and sequence searching was performed using PATRIC and NCBI, predicted secreted genes generated using SignalP and SecretomeP (see Methods).

expression of the $\beta$-oxidation genes is absent - i.e. it might be potentially constitutively expressed. On the other hand, SFB21 also contained multiple copies of FadE, which may indicate the ability to process FAs of a broad range. Both of the Serratia species contained multiple fadD genes encoding the Acyl-CoA synthetase (FadD), which activates the fatty acid for entrance into the $\beta$-oxidation cycle, but which most likely encode enzymes with specificities for small, medium or long-chain fatty acids, which may enable more efficient transport of a range of fatty acids into the cell.

\section{Growth of SFB isolates on FOGs}

The five strains were then tested for their ability to grow in synthetic wastewater with either addition of FOG ( $1 \%$ olive oil) or FOG plus acetate $\left(14 \mathrm{mM}, 0.81 \mathrm{mg} \mathrm{m}^{-1}\right)$. The strains were grown at $25 \mathrm{C}$ in a Tecan Sunrise with horizontal shaking with $\mathrm{OD}_{600}$ measurements taken at $30 \mathrm{~min}$ intervals, and all wells having respective control wells containing appropriate media blanks for each condition, which were subtracted from the culture positive wells to rule out any emulsification or precipitation effects. As shown in Fig. 4 we incubated the cultures for $72 \mathrm{~h}$ with all strains displaying multiphasic growth 

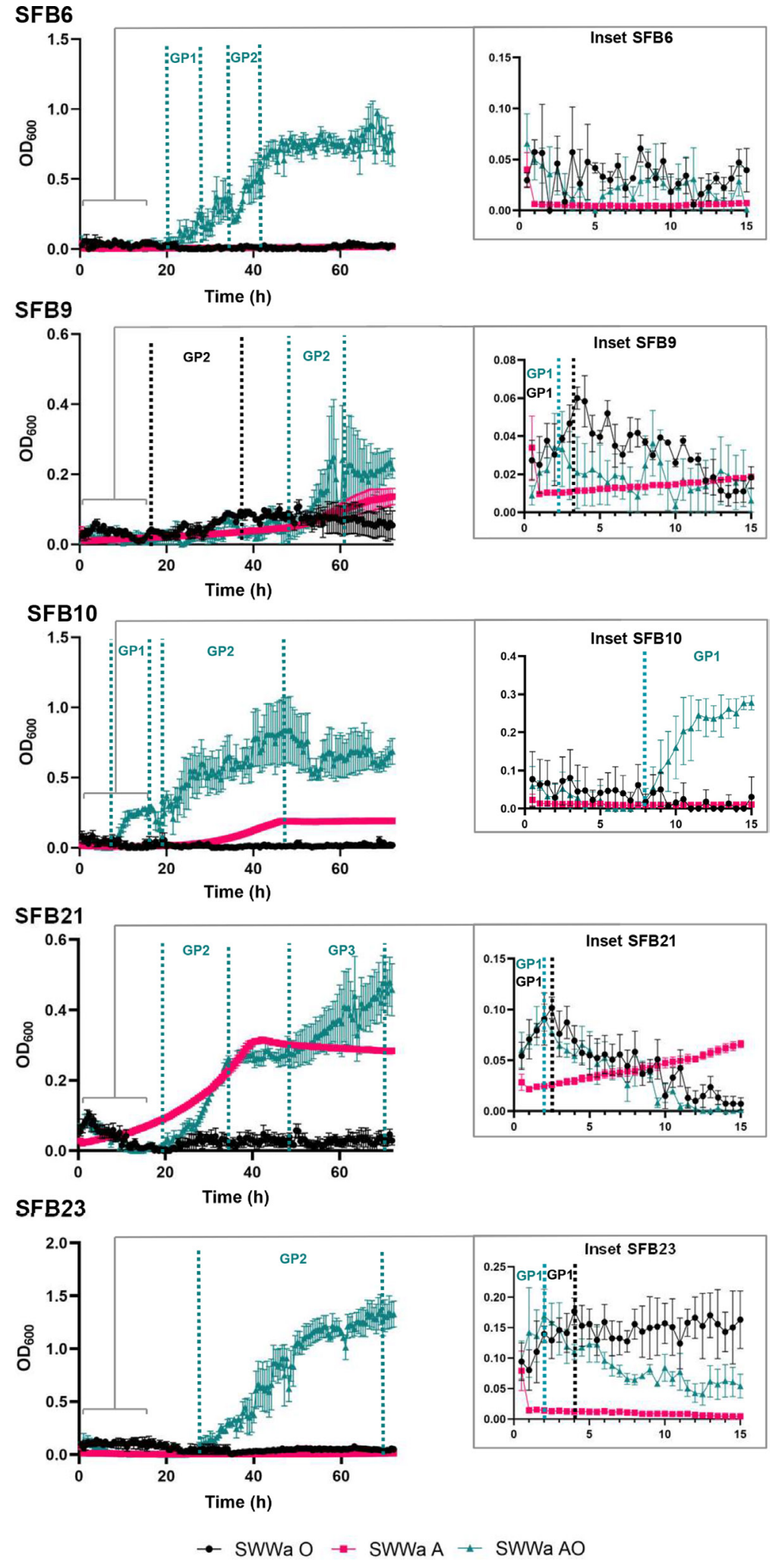

Fig. 4. Growth curves of the five isolates in SWWa with olive oil (SWWa +oil, black), acetic acid (SWWa A, magenta) or both olive oil and acetic acid (SWWa A+oil, teal). Vertical lines in the relevant colours and accompanied by GP1/2/3 depict putative alternate growth phases. The inset depicts the growth curve in the first $15 \mathrm{~h}$ in more detail. These are representative graphs of three technical replicates that were repeated three times. The $\mathrm{OD}_{600}$ is plotted with error bars showing standard error of the mean. 
patterns. In general, all isolates grew in the presence of acetate plus olive oil (Fig. 4, green) with varying peak $\mathrm{OD}_{600}(0.24$ to 1.4 ).

Isolates SFB 9, and 21 show growth in the presence of acetic acid only (magenta) with increased growth or altered growth rate in the presence of olive oil and acetic acid (teal, SFB9:0.24 vs 0.15 ; SFB21:0.48 vs 0.31), whereas no growth on acetate alone was observed for isolates SFB 6,10 and 23 (Fig. 4). Growth on olive oil only was generally low for all isolates compared to olive oil plus acetate but some growth was observed.

To illustrate this, we highlight the first $15 \mathrm{~h}$ in an inset in Fig. 4, revealing that for SFB 9, 10, 21 and 23 growth characteristics are different in the first $8 \mathrm{~h}$ as compared to extended $72 \mathrm{~h}$ incubation (Fig. 4 insets). For example, analysing growth on oil, cultures displayed a short rapid period of growth within the first $5 \mathrm{~h}$ with $\mathrm{OD}_{600}$ reaching around $0.06-0.18$ for SFB 9, 10, 21 and 23 (Fig. 4, insets GP1, black), which tails off, except for SFB9, which maintains slow growth on oil for $50 \mathrm{~h}$ (GP2, black). In the case of the acetate +oil cultures, all strains display a bi- or even tri-phasic growth pattern, where initial and subsequent growth periods are followed by periods of potential quiescence or adaptation before $\mathrm{OD}_{600}$ rises again, illustrated in Fig. 4 (GP2/3, teal), with isolate SFB21 a prime example. These data indicate a potential switch in growth modes/substrate or accumulation of toxic compounds in the cultures or possibly indicating alternating carbon sources that might arise from sequential FOG degradation - though this would need further investigation.

Taken together these data indicate the ability of these strains to grow in synthetic wastewater both in the presence of the FOG substrate olive oil but that they require acetate to boost growth to higher levels.

\section{S rDNA microbiome analysis of Fatberg site}

In order to characterize the microbial community present and ascertain if representatives of our isolates were present at the FOG blockage sites or might even be dominant, $16 \mathrm{~S}$ rDNA sequencing was carried out to determine the resident environmental bacterial microflora. In total, three swabs were taken from FOG deposits at site 1 and site 2 and the DNA extracted from the swabs using DNeasy PowerSoil kit and the V3/V4 variable regions were sequenced [commercially at MrDNA using primer set 341 F/ 806R [25]; on an Illumina MiSeq (MR DNA, Shallowater, TX, USA]. In these samples an average of 285 OTUs (+/-67 SD) were detected (Table S3).

The genus level data representing genera present at $0.5 \%$ of total reads or above for each sample are shown in Fig. 5a, and the top 75 most frequent genera detected of the total genus reads displayed by heatmap (Fig. 5b). Alpha diversity analysis of the samples (Simpson's index) is shown in Fig. 5c and varies between the sample groups, but the significance of the analysis is limited by the small sample size. A principal coordinates' analysis (Jansen-Shannon PCoA; Fig. 5d) does not show significant clustering of samples between sites 1 and 2, but does highlight that sample site 1 samples cluster together closely, however, sample site 2 has a larger diversity between its samples, with sample 2.2 clearly being more of an outlier in this analysis - again we add the caveat of small sample sizes here.

Our data reveal that these fatberg microbiota are composed of a diverse range of aerobic and anaerobic bacteria, with many of these genera containing species that are known to degrade lipids and commonly found in wastewater systems, e.g. Xanthomonas and Rhodobacter [53-55]. At site 1 , Xanthomonas is at the highest levels in all three samples (12-20\%) with the anaerobe Phascolarcobacterium (6-16\%) the next most abundant. Notably several other anaerobes are present, such as Bacteroides (2.9-5\%), Clostridium (2.2-3.4\%), Cloacibacterium (2.25-3.6\%) as well as Selenomonas, Prevotella, Parabacteriodes and Tannerella, which are all part of the anaerobic gut flora, but more broadly indicate the presence of anaerobic micro-environments in these FOG deposits. Notably the facultative genus Klebsiella (2.4-3.6\%) were also present at significant levels. Sample 2 is much more variable with Cloacibacterium at a very high level in sample $2.2(16.6 \%)$ but below $0.2 \%$ in sample 2.1 and 2.3 . The sequence with highest median value across the sample 2 datasets here is Acinetobacter (1.7-4.2\%), of note given our isolation data; and followed by the common environmental organism Hyphomicrobium (0.03-3.6\%). However, sample 2 has no dominant organism and a large quantity of sp. at low levels, indicating high diversity. Most of the genera mentioned above are common across both locations, with it notable that Acinetobacter, Klebsiella and Serratia as well as several environmental (Rhizobium), gut (Prevotella, Tannerella, Citrobacter and Bacteroides) were among these.

\section{DISCUSSION}

In this study, we set out to establish a pipeline for the isolation of potential fatberg-specific biodegraders and better understand the microbial environment of fatbergs to pave the way towards more target environment tailored products in the future.

As the first part of the study, we observed that the dominant fatty acid found in two separate fatberg deposits was palmitic acid ( $75 \%$ of the whole sample) - a finding that complements findings of other researchers in the USA and UK $[4,8,56]$.

We then developed a strategy that utilized high-throughput screening on Rhodamine agar plates, before secondary screening using a palmitate based colorimetric substrate (the major fatty acid in our samples) in a microplate format. In our growth experiments, isolates SFB9, 10 and 21 were able to grow on acetate only with isolates SFB6 and 23 showing no observable growth. All of the isolate growth seemed to be boosted by FOG (olive oil) inclusion in the media but only when acetate was present, with lower growth observable with olive oil as the only carbon source, thus highlighting their ability to potentially degrade and utilize FOG as a substrate for growth. This is reminiscent of diauxic growth that is 
(a)

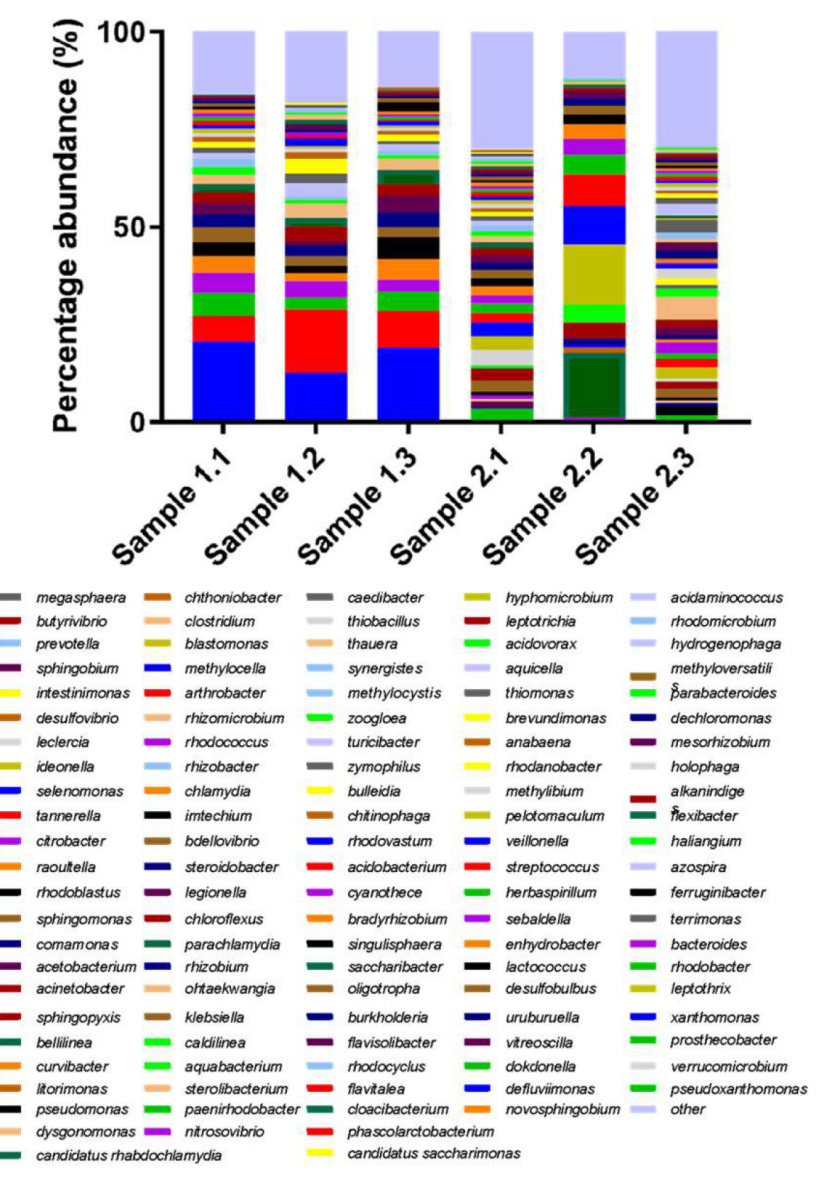

(c)

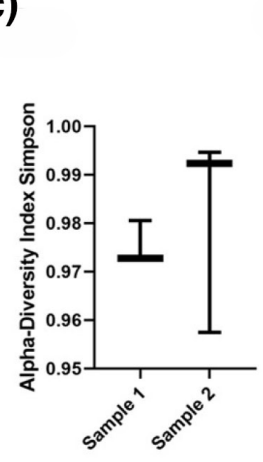

(d)

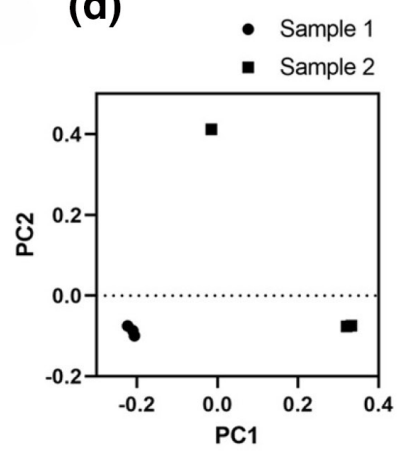

(b)
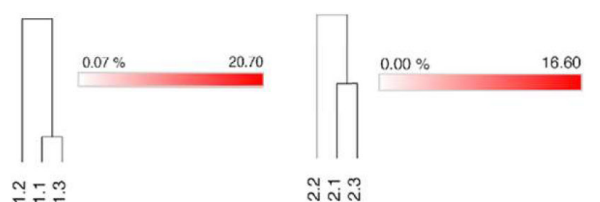

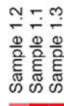

Genus

Xanthomonas

Xanthomonas
Phascolarctobacterium

Rhodobacter

Novosphingobium

Ferruginibacter

Rhizobium

Butyrivibrio

Klebsillla

Cloacibacterium

Clostridium

Sphingobium
Intestinimonas

Acidaminococcus

Megasphaera

Parabactero
Prevotella

Prevotellia
Desulfovibrio

Selenomonas

Rhodoblast
Leclercia

Ideonella

Acetobacterium

Tannerella

Citrobacter

Bellitinea

Comamonas

Paenirtodobacter

Raoultella

Sphingomonas
Acinetobacter

Acinetobacter

Turicibacter

Thauera

Acidovorax

Paracoccis

Bauldia

Synergistes

Zymophilus
Veillonella

Bulleidia

Bulleidia
Methanosaeta

Shigella

Eubacterium

Acetobacter

Macellibacteroides

Pleomorphomonas

Pseudomonas

Cloacibacillus

Lactobacillus

Roseomonas
Kluyvera

Methylosinus

Subdoligranulum

Aeromonas

Bosea

Flavonifractor

Dysgonomonas

Devosia

Xanthobacter

Ruminococcus

Enterobacter

Streptococcus

Legionella

Actinobaculum

Flexibacter

Ochrobactrum

Acidocella

Agrobacterium

Paludibacter

Pseudoxanthomonas

ูํำ

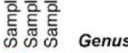

Cloacibacterium

Pelotomaculum

Veillonella

Acinetobacter

Sterolibacterium

Hyphomicrobium

Acidovorax

Sphingomonas

Rhodobacter

Herbaspirillum

Vyanothece

Acidobacterium

Acidobacterilum

Enhydrobacter

Enhydrobacter
Sphingopyxis

Prosthecobacter

Flavisolibacter

Rhodovastum

Thiomonas

Thiobacillus

Aquabacterium

Candidatus Saccharimonas

Burkholderia

Azospira

Bradyrhizobium

Legionella

Steroidobacter

Ferruginibacter

Lactococcus

Singulisphaer

Caedibacter

Methylocystis

Candidatus Rhabdochlamydi

Desulfobulbus

Methylocella

Uruburuella

Uruburuella
Sphingobium

Ontaekwangia

Vitreoscilla

Saccharibacter

Terrimonas

Desulfovibrio

Rhodomicrobium

Rhizobium

Comamonas

Rhodoblastus

Chthoniobacter

Clostridium
Chlamydia

Chlamydia

Rhodococcus
Blastomonas

Blastomonas
Bacteroides

Bacteroides
Bdellovibrio

Dechloromonas

Pseudomonas

Flavitalea

Litorimonas

Rhodanobacter

Holophaga

Imtechium

Arthrobacter

Citrobacter

Leptotrichia

Flexibacter

Mesorhizobium
Nitrosovibrio

Nitrosovibrio

Byssovorax

Byssovorax

Aquicella

Planctomyces

Fig. 5. (a) Bar charts showing the genus representing $>/=0.5 \%$ found by $16 \mathrm{~S}$ NGS sequencing from two FOG blockage sites in London sewers. (b) Heatmap displaying the top 75 most frequent genera based on the number of reads per sample (generated using Morpheus). (c) Simpson's index alpha diversity analysis of the sequencing data showing P-value: 0.66099; [T-test] statistic: -0.50313 . (d) Principal coordinate analysis (PCOA) based on Jensen-Shannon divergence distance showing similarities of samples from two fatberg sampling sites. PERMANOVA [f-value: 5.2369; R-squared: 0.56695; $P$-value $<0.1]$.

displayed by a range of organisms when switching carbon sources [57]. While other organisms often grow on multiple substrates, as was shown with Pseudomonas aeruginosa in the context of growth in tap water [58]. Finally, starvation in cultures can often influence metabolic capacity with glucose starved lipolytic B. licheniformis showing upregulation of lipid degrading pathways [59]. Considering our enrichment media contained acetate as well as FOG, a potential diauxie (e.g. SFB21) is perhaps not unexpected and our future studies will examine how varying acetate levels affects FOG-dependent 
growth and lipase production of isolates and indeed FOG levels in the media - assayed using Gravimetric methods. Notably, in preliminary studies (unpublished data), we observed a reduction in FOG levels in spent media when all five bacteria were grown in a consortium for $48 \mathrm{~h}$ with most degradation occurring later in the incubation cycle. One other aspect for development would be the enrichment strategy itself, with a reduction in acetate levels potentially allowing selection of organisms more dependent on FOG and allow assessment of the impact of alternative carbon sources in media on FOG metabolism or taking a limiting dilution approach as has been used in other environments to potentially improve recovery of organisms [60]. For example, there is evidence that some organisms upregulate lipase production in response to other carbon sources, with Acinetobacter iwoffii, which is related to SFB21, increasing lipase production in the presence of other hydrocarbons [61].

After genome sequencing, it was evident that our isolated strains contained a complement of predicted fatty acid degradation $(\mathrm{fad})$ genes, containing uptake and catabolism capability as well as a complement of lipases (Fig. 3b). These include lipase enzymes that are putatively secreted from the cell to hydrolyse TAGs (Triacylglycerols) into long-chain fatty acids (LCFAs) and a glycerol backbone. The LCFAs are transported into the cell using FadL transporter [62] and converted into coenzyme $\mathrm{A}(\mathrm{CoA})$ thioesters by the inner-membrane associated FadD, activating them [63]. This then enters the $\beta$-oxidation cycle where FadE converts acyl-CoA to enoylCoA and then FadBA tetramer catalyses the hydration, oxidation generating $\mathrm{NADH}$ and FADH2 and finally shortening of the acyl-CoA to give acetyl-CoA, which is processed by the TCA cycle [64]. The genomics of our strains identified two Serratia, two Klebsiella and a non-pathogenic Acinetobacter strain.

Of these strains, there have been several reports of stable lipase enzymes, with those from the Serratia strains the most well characterized, e.g. LipA from S. marcesens and SILipA form S. liquefaciens $[65,66]$. Similarly, interest in lipase from Klebsiella pneumoniae and oxytoca strains in relation to biodiesel production has also been reported with strains that were isolated from restaurant wastewater identified as degrading FOG [65-69]. In the case of Acinetobacter SFB21, this is part of a genus in which there is biotechnological interest with several novel lipases now discovered [70]. Notably SFB21 is most closely related to non-pathogenic Acinetobacter spp. that includes $A$. schindleri, bouvetii, Iwoffi and johnsonii and thus may have the potential for use in scale-up for deployment [71-73] N.B. a future focus of our work to determine whether SFB21 is a novel Acinetobacter spp. In fact, Acinetobacter strains, including SFB21 contain multiple copies of the fadE gene in their genomes, indicating a broad lipid catabolic capability [74], while Acinetobacter strains are known to grow well on acetate and palmitate [75-78], and to produce internal lipidbodies for energy storage [79, 80]. One future focus however will be to understand under what conditions the Fad genes are expressed, i.e. in the design of environmental deployment strategies within products- such as feedstocks.
Finally, Acinetobacter strains have been considered for biotechnological applications (including biofuel, pharmaceuticals and cosmetics) for the conversion of carbon substrates into useful oils such Triacylglycerols or in the form of Polyhydroxy Alkanoate polymers [81, 82]. This indicates the potential ability to use Acinetobacter strains (including SFB21) to remove FOG from a system, a capability that would be useful in FOG remediation applications and may suggest that future isolation strategies may consider targeting Acinetobacter strains more specifically using specialised media [83].

While the isolated strains have potential for remediation, one clear concern is potential pathogenic capability. This is a difficult conundrum since unsurprisingly these pathogenic faecal organisms are present in wastewater, however, it is in manufacturing processes and facilities that a potential problem arises with the manufacturing of potentially pathogenic Klebsiella and Serratia strains at scale. Therefore, given the non-pathogenic nature of Acinetobacter schindleri and bouvetii strains [77], like SFB21 here, it may be that future strategies should concentrate on the isolation of FOG degrading Acinetobacter strains for use in microbial consortia for FOG degradation as an alternative to Bacillus and Pseudomonas strains, although we acknowledge much of this work focuses on grease traps [84].

Finally, fatberg deposits are not composed solely of FOG, but are also made up of proteinaceous and carbohydrate substances [85]. Hence it is likely that any useful bioaddition strains or consortia should also have the ability to degrade a range of substrates. It is thus notable that the isolates in our study are also known for their ability to grow on a range of substrates and produce extracellular proteases, as seen in our genome sequences (not shown) but also in the literature [86].

As with any culture-based enrichment or selection strategy, the snapshot of organisms present is biassed by that enrichment media. Therefore, we also conducted 16S rDNA metagenomics of our two fatberg samples using Illumina-based sequencing. Firstly, the genus Acinetobacter and Klebsiella were found across all samples in the $16 \mathrm{~S}$ rDNA sequencing study performed (ranging from $0.36-4.2 \%$ and $0.04-3.6 \%$, respectively). In contrast, Serratia was only detected as a minor component of the fatberg microbiome $(<0.01 \%)$. Of note, the genera commonly used in FOG biological treatment products are reported to be Pseudomonas and Bacillus species [84, 87], but these contributed to less than $1 \%$ of the genera in our NGS screens. These data also revealed a diverse microbial community in these samples, on average around 300 potential species, many of which are commonly seen in wastewater samples (e.g. Xanthomonas, Fong and Tan [88]; Sphingomonas, Yoon et al. [89]; Rhodoacter, Hiraishi et al. [90]). In one of the sample site datasets (sample 1); there was dominance by the genus Xanthomonas (17.5\% of reads), an organism well known for its lipolytic properties in the context of plant pathogenesis [91]. In contrast, data from sample 2 were more variable, containing many spp. at much lower levels. Perhaps surprisingly both contained a significant number of obligate anaerobes, many members of 
the gut microflora (e.g. Bacteroides, Cloacibacterium, Prevotella) - however, our enrichment process would of course select against these and again may suggest future work should possibly take this into account. Future studies incorporating anaerobic selection conditions, alternative carbon sources (e.g. other carbohydrates, e.g. starch [85]) or limiting dilution approaches may improve recovery of species [60].

As outlined we chose five highly active strains for further characterization by genome sequencing and in growth experiments. Notably, all of our strains showed growth in the presence of olive oil as a FOG substrate in a synthetic wastewater medium (Fig. 4), which was boosted by inclusion of acetate indicating the potential to both degrade and utilize FOGs in the environment. While we have successfully isolated a range of FOG utilizing lipolytic strains, we are aware that none of our strains are well-adapted to growth solely on FOG, indicating that any potential application will need to ensure the presence of acetate. Alternatively, future enrichment strategies should attempt to isolate strains without acetate or attempt to adapt or evolve strains to improve FOG-dependent growth/degradation. Favourably, acetate levels are known to be significant in wastewater [48], suggesting that these organisms should survive in situ and that co-inoculation with acetate-based media may boost lipolysis. Carbon and nitrogen ratios have been identified as important for lipid degradation and so further investigation to determine performance and application of FOG degrading consortia would be important for future development [85].

\section{Conclusion}

In this study, we have increased understanding of the environment at FOG blockages and improved our understanding of microbial communities (microbiome) at fatberg sites, identifying for the first time the diverse range of organisms present.

Additionally, our isolation of a selection of lipolytic strains able to utilize FOGs as growth substrates highlights the potential of this approach of going to the environment for natural tailored solutions to solid FOG blockages, especially since they differ from current bioaddition strains in use (e.g. Bacillus strains). We consider these organisms, and especially Acinetobacter strain SFB 21 as having potential as part of a FOG degrading consortia, and preliminary unpublished work indicates that they are capable of reducing FOG content in domestic wastewater under laboratory conditions, and will be a focus of future work. We, therefore, have devised a strategy that could be applied to other wastewater situations to isolate potential FOG-treatment strains that are currently being taken forward and pave the way for the production of FOG-blockage tailored microbial consortia targeting solid deposits that may outperform current solutions.

\section{Funding information}

Genome sequencing was provided by MicrobesNG (http://www. microbesng.uk), which is supported by the UK Biotechnology and Biological Sciences Research Council (grant number BB/L024209/1). The authors would also like to acknowledge funding from the UK Engineering and Physical Sciences Research Council (EP/N010124/1) and the University of Sheffield Impact and Innovation Knowledge Exchange (156922).

\section{Acknowledgements}

The authors would like to acknowledge Thames Water for their professional support and sampling help and access for this work. Thanks also to Dr Esther Karunakaran (Department of Chemical and Biological Engineering, University of Sheffield, UK) who provided the synthetic wastewater media recipe designed in her laboratory by personal communication.

Conflicts of interest

The authors declare that there are no conflicts of interest.

\section{References}

1. He X, De Los FL, lii R, Ducoste JJ. Critical Reviews in Environmental Science and Technology A critical review of fat, oil, and grease (FOG) in sewer collection systems: Challenges and control A critical review of fat, oil, and grease (FOG) in sewer collection systems: Challenges and contro. Environ Sci Technol 2017;47:1191-1217.

2. Husain IAF, Alkhatib MF, Jammi MS, Mirghani MES, Bin Zainudin Z, et al. Problems, Control, and Treatment of Fat, Oil, and Grease (FOG): A Review. J Oleo Sci 2014;63:747-752.

3. Mattsson J, Hedström A, Viklander M, Blecken G-T. Fat, oil, and grease accumulation in sewer systems: comprehensive survey of experiences of scandinavian municipalities. J Environ Eng 2014;140:04014003.

4. Williams JB, Clarkson C, Mant C, Drinkwater A, May E. Fat, oil and grease deposits in sewers: Characterisation of deposits and formation mechanisms. Water Res 2012;46:6319-6328.

5. Oxford English Dictionary. fatberg, n: Oxford English Dictionary; (2021.). https://www.oed.com/view/Entry/59326664? redirectedFrom=fatberg\#eid

6. Dominic CCS, Szakasits M, Dean LO, Ducoste JJ. Understanding the spatial formation and accumulation of fats, oils and grease deposits in the sewer collection system. Water Sci Technol 2013;68:1830-1836.

7. Gurd C, Jefferson B, Villa R. Characterisation of food service establishment wastewater and its implication for treatment. J Environ Manage 2019:252:109657.

8. He X, lasmin M, Dean LO, Lappi SE, Ducoste JJ, et al. Evidence for Fat, Oil, and Grease (FOG) Deposit Formation Mechanisms in Sewer Lines. Environ Sci Technol 2011;45:4385-4391.

9. BBC News. "Monster" fatberg found blocking east London sewer - BBC News; 2017. https://www.bbc.co.uk/news/uk-englandlondon-41238272 [accessed 19.8.19].

10. Gross MA, Jensen JL, Gracz HS, Dancer J, Keener KM. Evaluation of physical and chemical properties and their interactions in fat, oil, and grease (FOG) deposits. Water Res 2017;123:173-182.

11. Southern Water. The impact of fat, oil and grease; 2018. https:// www.southernwater.co.uk/impact-of-fat-oil-grease

12. Wallace T, Gibbons D, O'Dwyer M, Curran TP. International evolution of fat, oil and grease (FOG) waste management - A review. J Environ Manage 2017;187:424-435.

13. Eljaiek-Urzola M, Romero-Sierra N, Segrera-Cabarcas L, Valdelamar-Martínez D, Quiñones-Bolaños É, et al. Oil and grease as a water quality index parameter for the conservation of marine biota. Water 2019:11:856.

14. Nieuwenhuis E, Post J, Duinmeijer A, Langeveld J, Clemens F. Statistical modelling of Fat, Oil and Grease (FOG) deposits in wastewater pump sumps. Water Res 2018;135:155-167.

15. He X, de los Reyes FL III, Leming ML, Dean LO, Lappi SE, et al. Mechanisms of Fat, Oil and Grease (FOG) deposit formation in sewer lines. Water Research 2013;47:4451-4459

16. Bielefeldt A, Gutierrez-Padilla MGD, Ovtchinnikov S, Silverstein J, Hernandez M. Bacterial kinetics of sulfur oxidizing bacteria and their biodeterioration rates of concrete sewer pipe samples. $J$ Environ Eng 2010;136:731-738. 
17. Győrik M, Ajtony Z, Dóka O, Alebic-Juretić A, Bicanic D, et al. Determination of free fatty acids in cooking oil: traditional spectrophotometry and optothermal window assay. Instrum Sci Technol 2006;34:119-128.

18. Quevatre C. War on fatbergs: Can this 21st Century peril be blitzed. BBC News Online, 2019.

19. Tzirita M, Papanikolaou S, Quilty B. Degradation of fat by a bioaugmentation product comprising of Bacillus spp. before and after the addition of a Pseudomonas sp. Eur J Lipid Sci Technol 2018;120:1700264.

20. Yun Z, Yun GH, Lee HS, Yoo TU. The variation of volatile fatty acid compositions in sewer length, and its effect on the process design of biological nutrient removal. Water Sci Technol 2013;67:2753-2760.

21. Kouker G, Jaeger KE. Specific and sensitive plate assay for bacterial lipases. Appl Environ Microbiol 1987;53:211-213.

22. Kapoore RV. Mass spectrometry based hyphenated techniques for microalgal and mammalian metabolomics. University of Sheffield, 2014.

23. Kapoore RV, Huete-Ortega M, Day JG, Okurowska K, Slocombe SP, et al. Effects of cryopreservation on viability and functional stability of an industrially relevant alga. Sci Rep 2019;9.

24. Pandhal J, Choon W, Kapoore R, Russo D, Hanotu J, et al. Harvesting environmental microalgal blooms for remediation and resource recovery: a laboratory scale investigation with economic and microbial community impact assessment. Biology 2017;7:4

25. Takahashi S, Tomita J, Nishioka K, Hisada T, Nishijima M, et al. Development of a prokaryotic universal primer for simultaneous analysis of Bacteria and Archaea using next-generation sequencing. PLoS One 2014:9:e105592.

26. Cole JR, Wang Q, Fish JA, Chai B, McGarrell DM, et al. Ribosomal Database Project: data and tools for high throughput rRNA analysis. Nucl Acids Res 2014;42:D633-D642.

27. Coordinators NR. Database resources of the National Center for Biotechnology Information. Nucleic Acids Res 2016:44:D7-D19.

28. MORPHEUS. Versatile matrix visualization and analysis software; 2020. https://software.broadinstitute.org/morpheus/

29. Arndt D, Xia J, Liu Y, Zhou Y, Guo AC, et al. METAGENassist: A comprehensive web server for comparative metagenomics. Nucleic Acids Res 2012;40:W88-95.

30. Chong J, Liu P, Zhou G, Xia J. Using MicrobiomeAnalyst for comprehensive statistical, functional, and meta-analysis of microbiome data. Nat Protoc 2020;15:799-821.

31. Dhariwal A, Chong J, Habib S, King IL, Agellon LB, et al. MicrobiomeAnalyst: a web-based tool for comprehensive statistical, visual and meta-analysis of microbiome data. Nucleic Acids Res 2017:45:W180-W188.

32. Bolger AM, Lohse M, Usadel B. Trimmomatic: A flexible trimmer for Illumina sequence data. Bioinformatics 2014;30:2114-2120.

33. Bankevich A, Nurk S, Antipov D, Gurevich AA, Dvorkin M, et al. SPAdes: A new genome assembly algorithm and its applications to single-cell sequencing. J Comput Biol 2012;19:455-477.

34. Seemann T. Prokka: Rapid prokaryotic genome annotation. Bioinformatics 2014:30:2068-2069.

35. Seemann T. VBC (Victorian Bioinformatics Consortium) - BARRNAP; 2014. http://www.vicbioinformatics.com/software.barrnap.shtml

36. Edgar RC. MUSCLE: Multiple sequence alignment with high accuracy and high throughput. Nucleic Acids Res 2004:32:1792-1797.

37. Stamatakis A. RAxML version 8: a tool for phylogenetic analysis and post-analysis of large phylogenies. Bioinformatics 2014;30:1312-1313.

38. Stamatakis A, Hoover P, Rougemont J. A rapid bootstrap algorithm for the RAxML web servers. Syst Biol 2008;57:758-771.

39. Okonechnikov K, Golosova O, Fursov M, UGENE team. Unipro UGENE: a unified bioinformatics toolkit. Bioinformatics 2012;28:1166-1167.
40. Davis JJ, Wattam AR, Aziz RK, Brettin T, Butler R, et al. The PATRIC Bioinformatics Resource Center: expanding data and analysis capabilities. Nucleic Acids Res 2020;48:D606-D612.

41. Marçais G, Delcher AL, Phillippy AM, Coston R, Salzberg SL, et al. MUMmer4: A fast and versatile genome alignment system. PLOS Comput Biol 2018;14:e1005944.

42. Wood DE, Salzberg SL. Kraken: Ultrafast metagenomic sequence classification using exact alignments. Genome Biol 2014:15:R46.

43. Almagro Armenteros JJ, Tsirigos KD, Sønderby CK, Petersen TN, Winther 0 , et al. SignalP 5.0 improves signal peptide predictions using deep neural networks. Nat Biotechnol 2019;37:420-423.

44. Bendtsen JD, Kiemer L, Fausbøll A, Brunak S. Non-classical protein secretion in bacteria. BMC Microbiol 2005;5:58.

45. Johnson M, Zaretskaya I, Raytselis Y, Merezhuk Y, McGinnis S, et al. NCBI BLAST: a better web interface. Nucleic Acids Res 2008:36:W5-9.

46. Wattam AR, Davis JJ, Assaf R, Boisvert S, Brettin T, et al. Improvements to PATRIC, the all-bacterial Bioinformatics Database and Analysis Resource Center. Nucleic Acids Res 2017;45:D535-D542.

47. Glogauer A, Martini VP, Faoro H, Couto GH, Müller-Santos M, et al. Identification and characterization of a new true lipase isolated through metagenomic approach. Microb Cell Fact 2011;10:54.

48. Raunkjaer K, Nielsen PH, Hvitved-Jacobsen T. Acetate removal in sewer biofilms under aerobic conditions. Water Res 1997;31:2727-2736.

49. WaterTech. Industrial and Commercial Bacteria - Wastewater, Fat, Oil, Grease, Septic, Odor Control; 2019. https://www.watertechgroup. $\mathrm{com} /$ en/industrial-commercial-reverse-osmosis-ro-and-watertreatment-systems/water-potable-and-waste-water-chemicals/ bacteria-wastewater-fat-oil-grease-septic-odor-control

50. Slade AH, Thorn GJS, Dennis MA. The relationship between BOD:N ratio and wastewater treatability in a nitrogen-fixing wastewater treatment system. Water Sci Technol 2011;63:627-632.

51. Boskou D, Blekas G, Tsimidou M. Olive oil composition. In: Olive Oil: Chemistry and Technology. Elsevier Inc, 2006.

52. Kinoshita K, Sadanami K, Kidera A, Go N. Structural motif of phosphate-binding site common to various protein superfamilies: all-against-all structural comparison of protein-mononucleotide complexes. Protein Eng Des Sel 1999;12:11-14.

53. Mo Q, Liu A, Guo H, Zhang Y, Li M. A novel thermostable and organic solvent-tolerant lipase from Xanthomonas oryzae pv. oryzae YB103: screening, purification and characterization. Extremophiles 2016;20:157-165

54. Yamaoka Y, Takeno K, Shinkawa H, Noparatnaraporn N, Sasaki K. Isolation of a thermotolerant photosynthetic bacterium, Rhodobacter sphaeroides strain, NAT, and Its capacity for oil and chemical oxygen demand removal at high temperatures. Biosci Biotechnol Biochem 2008:72:1601-1603.

55. Yamaoka Y, Takeno K, Noparatnaraporn N, Sasaki KEN. Oil Degradation Using a Photosynthetic Bacterium Rhodobacter sphaeroides S. Japanese Journal of Water Treatment Biology 2008;44:29-39.

56. Keener KM, Ducoste JJ, Holt LM. Properties Influencing Fat, Oil, and Grease Deposit Formation. Water Environ Res 2008;80:2241-2246.

57. Chu D, Barnes DJ. The lag-phase during diauxic growth is a trade-off between fast adaptation and high growth rate. Sci Rep 2016;6:1-15.

58. van der Kooij D, Oranje JP, Hijnen WA. Growth of Pseudomonas aeruginosa in Tap Water in Relation to Utilization of Substrates at Concentrations of a Few Micrograms per Liter. Appl Environ Microbiol 1982:44:1086-1095.

59. Voigt B, Hoi LT, Jürgen B, Albrecht D, Ehrenreich A, et al. The glucose and nitrogen starvation response of Bacillus licheniformis. Proteomics 2007;7:413-423.

60. Bai Y, Müller DB, Srinivas G, Garrido-Oter R, Potthoff E, et al. Functional overlap of the Arabidopsis leaf and root microbiota. Nature 2015;528:364-369.

61. Breuil C, Shindler DB, Sijher JS, Kushner DJ. Stimulation of lipase production during bacterial growth on alkanes. J Bacteriol 1978;133:601-606. 
62. van den Berg B. The FadL family: unusual transporters for unusual substrates. Curr Opin Struct Biol 2005;15:401-407.

63. Pech-Canul Á, Nogales J, Miranda-Molina A, Álvarez L, Geiger O, et al. FadD is required for utilization of endogenous fatty acids released from membrane lipids. J Bacteriol 2011;193:6295-6304.

64. Fujita Y, Matsuoka H, Hirooka K. Regulation of fatty acid metabolism in bacteria. Mol Microbiol 2007;66:829-839.

65. Akatsuka H, Kawai E, Omori K, Komatsubara S, Shibatani T, et al. The lipA gene of Serratia marcescens which encodes an extracellular lipase having no N-terminal signal peptide. J Bacteriol 1994;176:1949-1956.

66. Yao H, Yu S, Zhang L, Zuo K, Ling H, et al. Isolation of a Novel Lipase Gene from Serratia liquefaciens S33 DB-1. Functional Expression in Pichia pastoris and its Properties Mol Biotechnol 2008;38:99-107.

67. Mu Y, Xiu Z-L, Zhang D-J. A combined bioprocess of biodiesel production by lipase with microbial production of 1,3-propanediol by Klebsiella pneumoniae. Biochem Eng J 2008:40:537-541.

68. Nwaguma IV, Chikere CB, Okpokwasili GC. Isolation, characterization, and application of biosurfactant by Klebsiella pneumoniae strain IVN51 isolated from hydrocarbon-polluted soil in Ogoniland, Nigeria. Bioresour Bioprocess 2016:3:40.

69. Odeyemi AT, Aderiye BI, Bamidele OS. Lipolytic Activity of some Strains of Klebsiella, Pseudomonas and Staphylococcus Spp. from Restaurant Wastewater and Receiving Stream J Microbiology Res 2013:3:43-52.

70. Snellman EA, Colwell RR. Acinetobacter lipases: Molecular biology, biochemical properties and biotechnological potential. J Ind Microbiol Biotechnol 2004;31:391-400.

71. Batumalaie K, Khalili E, Mahat NA, Huyop F, Wahab RA. Biophysical characterization of a recombinant lipase KV1 from Acinetobacter haemolyticus in relation to $\mathrm{pH}$ and temperature. Biochimie 2018;152:198-210.

72. Gupta KK, Nigam A, Jagtap S, Krishna R. Scale-up and inhibitory studies on productivity of lipase from Acinetobacter radioresistens PR8. J Biosci Bioeng 2017;124:150-155.

73. Ran C, He S, Yang Y, Huang L, Zhou Z. A novel lipase as aquafeed additive for warm-water aquaculture. PLoS One 2015;10:e0132049.

74. Tani A, Ishige T, Sakai Y, Kato N. Two acyl-CoA dehydrogenases of Acinetobacter sp. strain $\mathrm{M}-1$ that uses very long-chain n-alkanes. J Biosci Bioeng 2002;94:326-329.

75. Fixter LM, Nagi MN, Mccormack JG, Fewson CA. Structure, distribution and function of wax esters in Acinetobacter calcoaceticus. Microbiology 1986;132:3147-3157.

76. Sampson KL, Finnerty WR. Regulation of fatty acid biosynthesis in the hydrocarbon oxidizing microorganism,Acinetobacter sp. Arch Microbiol 1974;99:203-220.

77. Sigala J-C, Suárez BP, Lara AR, Borgne SL, Bustos P, et al. Genomic and physiological characterization of a laboratory-isolated Acinetobacter schindleri ACE strain that quickly and efficiently catabolizes acetate. Microbiology (Reading) 2017;163:1052-1064.
78. Singer ME, Finnerty WR. Fatty aldehyde dehydrogenases in Acinetobacter sp. strain H01-N: role in hexadecanol metabolism. J Bacteriol 1985:164:1011-1016.

79. Alvarez HM, Steinbüchel A. Triacylglycerols in prokaryotic microorganisms. Appl Microbiol Biotechnol 2002;60:367-376.

80. Wältermann M, Hinz A, Robenek H, Troyer D, Reichelt R, et al. Mechanism of lipid-body formation in prokaryotes: how bacteria fatten up. Mol Microbiol 2004:55:750-763.

81. Santala S, Efimova E, Kivinen V, Larjo A, Aho T, et al. Improved triacylglycerol production in Acinetobacter baylyi ADP1 by Metabolic Engineering. Microb Cell Fact 2011;10:36.

82. Santala S, Efimova E, Santala V. Dynamic decoupling of biomass and wax ester biosynthesis in Acinetobacter baylyi by an autonomously regulated switch. Metab Eng Commun 2018;7:e00078.

83. Jawad A, Hawkey PM, Heritage J, Snelling AM. Description of Leeds Acinetobacter medium, a new selective and differential medium for isolation of clinically important Acinetobacter spp., and comparison with Herellea agar and Holton's agar. J Clin Microbiol 1994:32:2353-2358.

84. Tzirita M, Papanikolaou S, Quilty B. A study of the suitability of three commercial bioaugmentation products for use in grease traps. Biomass Conv Bioref 2019;11:907-924.

85. Gurd C, Villa R, Jefferson B. Understanding why fat, oil and grease (FOG) bioremediation can be unsuccessful. J Environ Manage 2020;267:110647.

86. Yuan L, Sadiq FA, Liu T-J, Li Y, Gu J-S, et al. Spoilage potential of psychrotrophic bacteria isolated from raw milk and the thermostability of their enzymes. J Zhejiang Univ Sci B 2018;19:630-642.

87. Shamaan NA, Shukor MYA, Ahmadun FR, Abdullah N. Bacteria for the Degradation and Modification of Fats, Oils and Grease. 2008.

88. Fong KPY, Tan HM. Isolation of a microbial consortium from activated sludge for the biological treatment of food waste. World J Microbiol Biotechnol 2000;16:441-443.

89. Yoon JH, Park S, Kang SJ, Kim W, Oh TK. Sphingomonas hankookensis sp. nov., isolated from wastewater. Int J Syst Evol Microbiol 2009:59:2788-2793.

90. Hiraishi A, Muramatsu K, Urata K. Characterization of new denitrifying Rhodobacter strains isolated from photosynthetic sludge for wastewater treatment. J Ferment Bioeng 1995;79:39-44.

91. Tamir-Ariel D, Rosenberg T, Navon N, Burdman S. A secreted lipolytic enzyme from Xanthomonas campestris pv. vesicatoria is expressed in planta and contributes to its virulence. Mol Plant Pathol 2012;13:556-567.

92. Jimenez-Diaz L, Caballero A, Segura A. Pathways for the Degradation of Fatty Acids in Bacteria. In: Aerobic Utilization of Hydrocarbons, Oils and Lipids. Cham: Springer International Publishing, 2017

Edited by: S. P Diggle and G. Dey

\section{Five reasons to publish your next article with a Microbiology Society journal}

1. The Microbiology Society is a not-for-profit organization.

2. We offer fast and rigorous peer review - average time to first decision is 4-6 weeks.

3. Our journals have a global readership with subscriptions held in research institutions around the world.

4. $80 \%$ of our authors rate our submission process as 'excellent' or 'very good'.

5. Your article will be published on an interactive journal platform with advanced metrics.

Find out more and submit your article at microbiologyresearch.org. 